Review began $01 / 27 / 2022$ Review ended 01/30/2022 Published 02/04/2022

(c) Copyright 2022

Gonzalez et al. This is an open access article distributed under the terms of the Creative Commons Attribution License CC BY 4.0., which permits unrestricted use, distribution, and reproduction in any medium, provided the original author and source are credited.

\section{Central Diabetes Insipidus Masked by Uncontrolled Diabetes Mellitus: A Challenging Case Managed With Indapamide}

Eyleen Gonzalez ${ }^{1}$, Lorena Nuñez ${ }^{2}$, Yavelkis Perez ${ }^{2}$, Indira Atencio ${ }^{2}$, Alex Pineda ${ }^{2}$, Myron Miller ${ }^{3,4}$, Stanley M. Chen Cardenas 1, 2, 4

1. Medicine, Facultad de Medicina, Universidad de Panama, Panama, PAN 2. Internal Medicine, Hospital Nicolas A. Solano, West Panama, PAN 3. Medicine, Sinai Hospital of Baltimore, Baltimore, USA 4. Endocrinology, Diabetes and Metabolism, Johns Hopkins University School of Medicine, Baltimore, USA

Corresponding author: Stanley M. Chen Cardenas, schencardenas@gmail.com

\section{Abstract}

A 44-year-old man with a history of traumatic brain injury (TBI) presented to the emergency room (ER) with diabetic ketoacidosis (DKA). After resolution of DKA, the patient had persistent polyuria (up to $5.5 \mathrm{~L} / 24 \mathrm{~h}$ ) associated with low specific gravity (1.002-1.005) and severe hypernatremia (up to $186 \mathrm{mmol} / \mathrm{L}$ ) that led us to consider the possibility of central diabetes insipidus (DI). Due to the lack of desmopressin availability in our country, we managed the patient using indapamide. Polydipsia and polyuria in a patient with controlled diabetes mellitus (DM) should raise suspicion for alternative etiologies, including DI. Appropriate fluid management during hospitalization is critical to avoid life-threatening complications. TBI is an important cause of central DI and should be treated with desmopressin, an arginine-vasopressin (AVP) analog. In the absence of desmopressin, alternative options can help patients with central DI, including thiazides, carbamazepine, chlorpropamide, among others less studied.

Categories: Endocrinology/Diabetes/Metabolism, Internal Medicine, Neurosurgery

Keywords: desmopressin, diabetic ketoacidosis (dka), indapamide, thiazides, traumatic brain injury, hypernatremia, polyuria, diabetes insipidus

\section{Introduction}

Diabetic ketoacidosis (DKA) is a life-threatening complication of diabetes mellitus (DM) [1]. It occurs more commonly in type I DM but can present in type II DM when the insulin deficit is marked. DKA is characterized by polydipsia, polyuria, altered mental status, acidosis with electrolyte disturbances such as hyponatremia, pseudohyponatremia, or hypernatremia. Diabetes insipidus (DI) in contrast, is a disorder of water balance that occurs much less frequently than DM. DI can be classified as central when the problem is in the hypothalamic-pituitary region with insufficient production of arginine-vasopressin (AVP) and nephrogenic when the kidney does not respond to AVP [2-3]. Both forms of DI lead to a decrease in water reabsorption in the kidney resulting in hypo-osmolar polyuria. In the absence of thirst or fluid access, DI can lead to severe dehydration, hypernatremia, and death.

In both DKA and DI, polyuria and hypernatremia can occur, which can be challenging to manage if they coexist. We present a patient admitted with DKA who had persistent polyuria and hypernatremia despite correction of hyperglycemia. Due to the lack of desmopressin treatment for central DI in our low-resource healthcare setting, an inexpensive alternative medication resulted in successful treatment.

\section{Case Presentation}

A 44-year-old man with a history of a traumatic brain injury (TBI) after being hit by a car as a pedestrian suffered a left frontal and orbital fracture 11 months prior to presentation. He presented to the emergency room (ER) with one week of generalized weakness, worsening polyuria, polydipsia, and altered mental status. He was found to have DKA with a blood $\mathrm{pH}$ of 7.2 , bicarbonate of $5.9 \mathrm{mmol} / \mathrm{L}$, carbon dioxide 15 $\mathrm{mmHg}$, anion gap 25, glucose $484 \mathrm{~g} / \mathrm{dL}$, positive urinary ketones, and normal lactate. He was managed according to the DKA protocol.

Over the next $24 \mathrm{~h}$, his serum glucose was challenging to control, requiring up to 14 units of insulin per hour while fasting. At the same time despite persistent hyperglycemia, his serum sodium continued rising reaching a maximum corrected of $186 \mathrm{mmol} / \mathrm{L}$ (Figure 1). After DKA resolution, hypernatremia and excessive urination persisted ( $>3 \mathrm{~L} /$ day). Initially, it was thought that polyuria was due to glycosuria from uncontrolled diabetes. However, its persistence after glucose was $<180 \mathrm{mg} / \mathrm{dL}$, led us to consider alternative explanations. On reviewing his course, increased serum sodium level occurred rapidly in the setting of limited access to water and high urine output $(>400 \mathrm{~mL} / \mathrm{h}$ ) after DKA resolution. The specific gravity in his initial urinalysis was 1.020 with corrected serum sodium of $147 \mathrm{mmol} / \mathrm{L}$. Over the following day, the specific gravity decreased to 1.005 while corrected serum sodium reached $176 \mathrm{mmol} / \mathrm{L}$. Given the history of TBI and clinical 


\section{Cureus}

findings (Table 1 ) we considered the possibility of central DI. Our hospital unfortunately did not have the ability to measure urine osmolality or perform a pituitary MRI at the time. His calculated water deficit was up to $14 \mathrm{~L}$ and hypernatremia developed in $<48 \mathrm{~h}$. An aggressive hypotonic fluid replacement was started and as soon as the patient was awake he drank free water in response to his intact thirst mechanism. Additionally, given his progressive increase in urine output and severe hypernatremia, a total of four doses of five units sub-cutaneous (SQ) of vasopressin were administered during days two to four of admission resulting in a reduction in diuresis to as low as $75 \mathrm{~mL} / \mathrm{h}$, thus excluding the possibility of nephrogenic DI (Figure 1). The hospital course was complicated by transient electrolyte abnormalities such as hypokalemia, hypophosphatemia, and acute kidney injury (AKI) (Table 1). By day five to seven of admission, blood glucose was controlled and no DKA precipitant was found. He was fully awake and drinking large amounts of water to maintain the fluid balance close to neutral while serum sodium in the $150 \mathrm{~s}$.
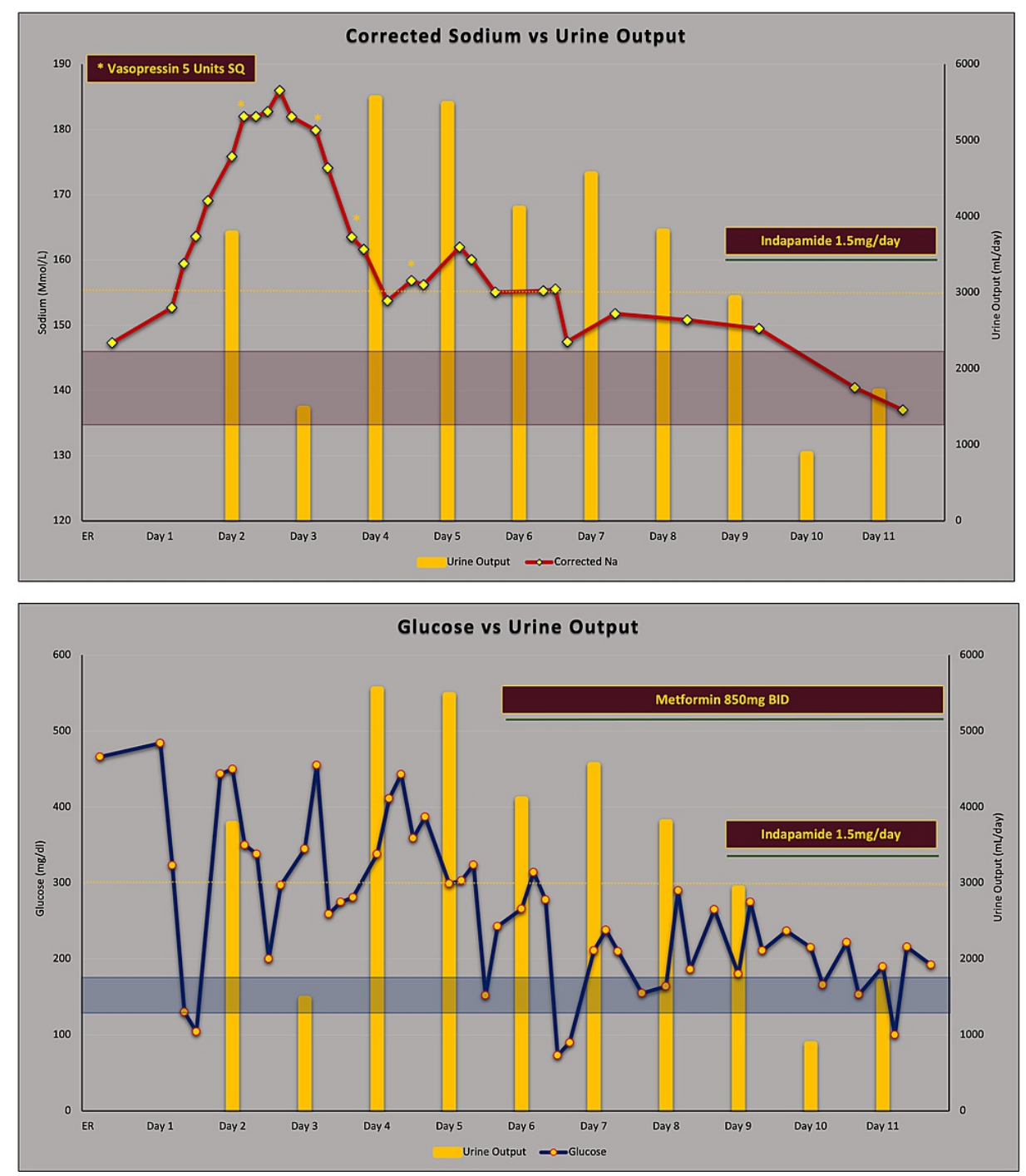

FIGURE 1: Corrected sodium, glucose, and urine output.

Description of the relationship between corrected sodium [formula: corrected sodium $=$ measured $\mathrm{Na}+[$ (glucose level -100$) \times 0.016$ ] and urine output (top). Relationship between glucose and urine output (bottom) associated with medications used in the patient (vasopressin, metformin, and indapamide) 


\section{Cureus}

\begin{tabular}{|c|c|c|c|}
\hline Laboratory & Reference range & Admission & Discharge \\
\hline Sodium (mmol/L) & 135-145 & 140 & 137 \\
\hline Glucose (mg/dL) & $70-100$ & 466 & 185 \\
\hline Corrected sodium & 135-145 & 147 & 138 \\
\hline Serum osmolality (mOsm/L) & 275-295 & 314 & 288 \\
\hline Creatinine $(\mathrm{mg} / \mathrm{dL})$ & $0.7-1.3$ & 1.06 & 0.7 \\
\hline BUN (mg/dL) & $8-20$ & 23 & 6 \\
\hline Potassium (mmol/L) & $3.5-5.1$ & 3.8 & 3.5 \\
\hline Bicarbonate $(\mathrm{mmol} / \mathrm{L})$ & $22-30$ & 5.9 & 28.9 \\
\hline Anion gap (mmol/L) & $8-16$ & 25 & 3 \\
\hline Calcium (mg/dL) & $8.6-10.3$ & 10.3 & 9.6 \\
\hline White blood cells $\left(10^{3} / \mathrm{uL}\right)$ & $4-10$ & 10.3 & 6.7 \\
\hline Neutrophils (\%) & $50-80$ & 81.2 & 59.6 \\
\hline Hemoglobin (g/dL) & 13-17 & 18.2 & 15.3 \\
\hline Hematocrit (\%) & $39-50$ & 52.6 & 42.4 \\
\hline Platelets (103/uL) & $140-400$ & 182 & 242 \\
\hline Urine specific gravity & $1.000-1.030$ & 1.020 & $1.003^{*}$ \\
\hline Glycosuria & Negative & $4+(>1000 \mathrm{mg} / \mathrm{dL})$ & Negative $^{*}$ \\
\hline Urine ketones & Negative & $3+$ & Negative $^{*}$ \\
\hline
\end{tabular}

\section{TABLE 1: Laboratories on admission and discharge.}

*Obtained two days prior to discharge with a sodium $146 \mathrm{mmol} / \mathrm{L}$

BUN, blood urea nitrogen

On planning for discharge, we found that desmopressin was not available in the country. Based on its efficacy in this setting, safety profile, accessibility, and low cost, we started treatment with indapamide 1.5 $\mathrm{mg}$ daily. Even though the patient was normotensive, he tolerated the drug well except for mild hypokalemia. His urine output decreased markedly from up to $5.5 \mathrm{~L}$ after DKA resolution to as low as $0.9 \mathrm{~L}$ per day by one day after starting indapamide (Figure 1). Serum sodium level decreased to $137 \mathrm{mmol} / \mathrm{L}$, specific gravity remained low at 1.003 while drinking water as per thirst. He was successfully discharged with insulin glargine 36 units/day, lispro 12 units before meals, metformin $850 \mathrm{mg}$ BID, indapamide $1.5 \mathrm{mg} /$ day, and oral potassium supplements.

On further questioning, he recalled that after the motor vehicle accident he was always thirsty and urinating large volumes at home that got progressively worse until the current hospitalization. He was discharged with plans for follow-up evaluation for other potential hypothalamic-pituitary hormonal deficiencies from the TBI. The patient was involved in the decision-making process and was very pleased with the results. Three weeks after discharge, he reported a resolution of polyuria and a normal water intake.

\section{Discussion}

It is important to keep a broad differential diagnosis in patients with polyuria, particularly when unusual clinical features are present. In this young patient, severe dehydration evidenced by AKI,

hemoconcentration, and hypernatremia was associated with high instead of low urine output that persisted after DKA resolution. Polyuria can be due to solute diuresis where urine osmolality (Uosm) is $>300 \mathrm{mOsm} / \mathrm{kg}$ and can occur during hyperglycemia, recovery from acute renal failure, relief of urinary obstruction or exogenous solute load from enteral or parenteral nutrition, radiocontrast dyes, sodium, or mannitol. Polyuria can also result from water diuresis where Uosm is $<100 \mathrm{mOsm} / \mathrm{kg}$ and is caused by central or nephrogenic DI. A combination of both solute and water diuresis (mixed polyuria) can develop with 
Uosm between 100 and $300 \mathrm{mOsm} / \mathrm{kg}$ and could be due to partial DI or multifactorial [4-5]. The diuresis during uncontrolled DM is osmotic and independent from AVP; in fact, in DKA, AVP is elevated, granted that hypovolemia and nausea/vomiting are often present [6-7]. In our patient, the combination of hyperglycemia and central DI explains his initial excessive urination. After controlling hyperglycemia, central DI emerged as a more likely cause.

Desmopressin was unavailable, which allowed us to search for alternative treatments for partial central DI. Central DI is routinely treated with oral desamino-D-arginine-8-vasopresssin (DDAVP or desmopressin), a longer-acting and V2 receptor-specific AVP analog. This drug can also be prescribed as sublingual, intranasal, inhaled, subcutaneous, or IV. Prior to the availability of DDAVP in the late 1970s, alternative drugs such as chlorpropamide, carbamazepine, and thiazides were studied. Chlorpropamide is a long-acting first-generation sulfonylurea previously used to treat DM. Its antidiuretic properties were discovered in the 1960s when a patient with DI took the medication by mistake [8]. Chlorpropamide can increase AVP secretion and enhance its action in the kidney [9-10]. It is effective in reducing urine output and increasing urine osmolality in patients with DI. Doses between 125 and $1000 \mathrm{mg} /$ day have been used, but the risk of hypoglycemia is the limiting factor in using this drug $[8,11]$. Other sulfonylureas lack this antidiuretic effect. Also, metformin has reported antidiuretic effects [12]. It is possible that giving metformin to our patient contributed to his improvement. Carbamazepine and oxcarbazepine are voltage-gated sodium channel blockers used as antiepileptics. It appears that they can increase AVP secretion. More recently, direct action on the V2R-G-protein complex with increased AQ2 expression and resultant decreased water excretion independent from AVP has been described [13]. Prolongation of AVP half-life and resetting the AVP-osmotic hypothalamic threshold are potential mechanisms. Reports using carbamazepine in doses of 200-600 $\mathrm{mg} /$ day and 600-900 mg BID for oxcarbazepine have been documented [10, 14]. Thiazides are commonly used diuretics that block the sodium-chloride transporter in the distal tubule. Hydrochlorothiazide, indapamide, and chlorothiazide have been studied in DI [15]. Thiazides increase sodium excretion leading to volume contraction, reduction in glomerular filtration rate, increasing proximal sodium, and water reabsorption. It is believed that this results in less solute and water delivery to the distal tubules and collecting ducts reducing the urine output, therefore, AVP independent [16]. A study using indapamide demonstrated a reduction in urine output of about $40 \%$ together with an increase in urine and a decrease in serum osmolality comparable to chlorpropamide [17]. Non-steroidal anti-inflammatory drugs (NSAIDs) such as ibuprofen and indomethacin have shown a reduction in urine volume. Prostaglandin E2 (PGE2) is an inhibitor of the AVP effect, as well as a local vasodilator on the renal vasculature, therefore, inhibition of PGE2 by NSAIDs has an antidiuretic effect [10,18]. Clofibrate has also reported antidiuretic properties possibly by increasing the release of AVP [19]. Furthermore, acetaminophen, amitriptyline, vincristine, cyclophosphamide, diazoxide, valproic acid, selective serotonin reuptake inhibitors (SSRIs), and selective serotonin reuptake inhibitors (SNRIs) have reported antidiuretic effects and can produce the syndrome of inappropriate antidiuretic hormone secretion [12].

We decided to use indapamide given its safety profile and availability in our institution [17]. Possibly this patient could maintain acceptable serum sodium levels with aggressive oral intake. However, if access to water is limited or severe illness is present, this may not be possible.

\section{Conclusions}

We consider it is essential for the clinician to know that for central DI when DDAVP is not available or the cost is a problem, other alternatives can be used to lower urine output, stimulate AVP release, or potentiate its renal effect. This is particularly relevant in low-resource healthcare settings. Polyuria and hypernatremia should raise suspicion for DI, even in patients with uncontrolled DM, particularly when a risk factor such as TBI is present, as in this case. The coexistence of DKA and DI is rare and could be potentially lethal if not properly recognized and managed.

\section{Additional Information \\ Disclosures}

Human subjects: Consent was obtained or waived by all participants in this study. Conflicts of interest: In compliance with the ICMJE uniform disclosure form, all authors declare the following: Payment/services info: All authors have declared that no financial support was received from any organization for the submitted work. Financial relationships: All authors have declared that they have no financial relationships at present or within the previous three years with any organizations that might have an interest in the submitted work. Other relationships: All authors have declared that there are no other relationships or activities that could appear to have influenced the submitted work.

\section{Acknowledgements}

We would like to thank Dr. Idelfonso Menacho, Dr. Nurith Thompson, and Dr. Alexander Xian for their help in caring for this patient.

\section{References}


1. Dhatariya KK, Glaser NS, Codner E, Umpierrez GE: Diabetic ketoacidosis. Nat Rev Dis Primers. 2020, 6:1-20. 10.1038/s41572-020-0165-1

2. Miller M, Dalakos T, Moses AM, Fellerman H, Streeten DH: Recognition of partial defects in antidiuretic hormone secretion. Ann Intern Med. 1970, 73:721-729. 10.7326/0003-4819-73-5-721

3. Christ-Crain M, Bichet DG, Fenske WK, Goldman MB, Rittig S, Verbalis JG, Verkman AS: Diabetes insipidus. Nat Rev Dis Primers. 2019, 5:1-20. 10.1038/s41572-019-0103-2

4. Bhasin B, Velez JC: Evaluation of polyuria: the roles of solute loading and water diuresis . Am J Kidney Dis. 2016, 67:507-511. 10.1053/j.ajkd.2015.10.021

5. Robertson GL: Differential diagnosis of polyuria. Annu Rev Med. 1988, 39:425-442. 10.1146/annurev.me.39.020188.002233

6. Bankir L, Bardoux P, Ahloulay M: Vasopressin and diabetes mellitus. Nephron. 2001, 87:8-18. 10.1159/000045879

7. Zerbe RL, Vinicor F, Robertson GL: Plasma vasopressin in uncontrolled diabetes mellitus. Diabetes. 1979, 28:503-508. 10.2337/diab.28.5.503

8. Arduino F, Ferraz FP, Rodrigues J: Antidiuretic action of chlorpropamide in idiopathic diabetes insipidus . J Clin Endocrinol Metab. 1966, 26:1325-1328. 10.1210/jcem-26-12-1325

9. Miller M, Moses AM: Mechanism of chlorpropamide action in diabetes insipidus . J Clin Endocrinol Metab. 1970, 30:488-496. 10.1210/jcem-30-4-488

10. Singer I, Oster JR, Fishman LM: The management of diabetes insipidus in adults . Arch Intern Med. 1997, 157:1293-1301. 10.1001/archinte.1997.00440330027003

11. Cushard WG Jr, Beauchamp CJ, Martin ND: Oral therapy of diabetes insipidus with chlorpropamide . Calif Med. 1971, 115:1-5.

12. Moses AM, Miller M: Drug-induced dilutional hyponatremia. N Engl J Med. 1974, 291:1234-1239. 10.1056/NEJM197412052912307

13. de Bragança AC, Moyses ZP, Magaldi AJ: Carbamazepine can induce kidney water absorption by increasing aquaporin 2 expression. Nephrol Dial Transplant. 2010, 25:3840-3845. 10.1093/ndt/gfq317

14. Abdallah B, Hodgins S, Landry D, O'shea M, Braden G: Oxcarbazepine therapy for complete central diabetes insipidus. Case Rep Nephrol Dial. 2018, 8:20-24. 10.1159/000485244

15. Crawford JD, Kennedy GC: Chlorothiazid in diabetes insipidus. Nature. 1959, 183:891-892. 10.1038/183891a0

16. Magaldi AJ: New insights into the paradoxical effect of thiazides in diabetes insipidus therapy . Nephrol Dial Transplant. 2000, 15:1903-1905. 10.1093/ndt/15.12.1903

17. Tetiker T, Sert M, Koçak M: Efficacy of indapamide in central diabetes insipidus . Arch Intern Med. 1999, 159:2085-2087. 10.1001/archinte.159.17.2085

18. Libber S, Harrison H, Spector D: Treatment of nephrogenic diabetes insipidus with prostaglandin synthesis inhibitors . J Pediatr. 1986, 108:305-311. 10.1016/s0022-3476(86)81010-1

19. Moses AM, Howanitz J, van Gemert M, Miller M: Clofibrate-induced antidiuresis. J Clin Invest. 1973, 52:535542. 10.1172/JCI107213 